\title{
An Algorithm for Finding a Common Solution for a System of Mixed Equilibrium Problem, Quasivariational Inclusion Problem, and Fixed Point Problem of Nonexpansive Semigroup
}

\author{
M. Liu, S. S. Chang, and P. Zuo \\ Department of Mathematics, Yibin University, Yibin, Sichuan 644007, China \\ Correspondence should be addressed to M. Liu, liuminybsc@yahoo.com.cn and \\ S. S. Chang, changss@yahoo.cn
}

Received 31 March 2010; Accepted 8 June 2010

Academic Editor: Vy Khoi Le

Copyright (C) 2010 M. Liu et al. This is an open access article distributed under the Creative Commons Attribution License, which permits unrestricted use, distribution, and reproduction in any medium, provided the original work is properly cited.

We introduce a hybrid iterative scheme for finding a common element of the set of solutions for a system of mixed equilibrium problems, the set of common fixed point for nonexpansive semigroup, and the set of solutions of the quasi-variational inclusion problem with multivalued maximal monotone mappings and inverse-strongly monotone mappings in Hilbert space. Under suitable conditions, some strong convergence theorems are proved. Our results extend some recent results announced by some authors.

\section{Introduction}

Throughout this paper we assume that $H$ is a real Hilbert space, and $C$ is a nonempty closed convex subset of $H$.

In the sequel, we denote the set of fixed points of $S$ by $F(S)$.

A bounded linear operator $A: H \rightarrow H$ is said to be strongly positive, if there exists a constant $\bar{\gamma}$ such that

$$
\langle A x, x\rangle \geq \bar{r}\|x\|^{2}, \quad \forall x \in H .
$$

Let $B: H \rightarrow H$ be a single-valued nonlinear mapping and $M: H \rightarrow 2^{H}$ a multivalued mapping. The "so-called" quasi-variational inclusion problem (see, Chang $[1,2]$ ) is to find an $u \in H$ such that

$$
\theta \in B(u)+M(u)
$$


A number of problems arising in structural analysis, mechanics, and economics can be studied in the framework of this kind of variational inclusions (see, e.g., [3]).

The set of solutions of variational inclusion (1.2) is denoted by $\operatorname{VI}(H, B, M)$.

\section{Special Case}

If $M=\partial \delta_{C}$, where $C$ is a nonempty closed convex subset of $H$, and $\delta_{C}: H \rightarrow[0, \infty)$ is the indicator function of $C$, that is,

$$
\delta_{C}= \begin{cases}0, & x \in C, \\ +\infty, & x \notin C,\end{cases}
$$

then the variational inclusion problem (1.2) is equivalent to find $u \in C$ such that

$$
\langle B(u), v-u\rangle \geq 0, \quad \forall v \in C
$$

This problem is called Hartman-Stampacchia variational inequality problem (see, e.g., [4]). The set of solutions of (1.4) is denoted by $\operatorname{VI}(C, B)$.

Recall that a mapping $B: H \rightarrow H$ is called $\alpha$-inverse strongly monotone (see [5]), if there exists an $\alpha>0$ such that

$$
\langle B x-B y, x-y\rangle \geq \alpha\|B x-B y\|^{2}, \quad \forall x, y \in H
$$

A multivalued mapping $M: H \rightarrow 2^{H}$ is called monotone, if for all $x, y \in H, u \in M x$, and $v \in M y$, then it implies that $\langle u-v, x-y\rangle \geq 0$. A multivalued mapping $M: H \rightarrow 2^{H}$ is called maximal monotone, if it is monotone and if for any $(x, u) \in H \times H$

$$
\langle u-v, x-y\rangle \geq 0, \quad \forall(y, v) \in \operatorname{Graph}(M)
$$

(the graph of mapping $M$ ) implies that $u \in M x$.

Proposition 1.1 (see [5]). Let $B: H \rightarrow H$ be an $\alpha$-inverse strongly monotone mapping, then

(a) $B$ is a $1 / \alpha$-Lipschitz continuous and monotone mapping;

(b) if $\lambda$ is any constant in $(0,2 \alpha]$, then the mapping $I-\lambda B$ is nonexpansive, where $I$ is the identity mapping on $\mathrm{H}$.

Let $\Theta: C \times C \rightarrow R$ be an equilibrium bifunction (i.e., $\Theta(x, x)=0$, for all $x \in C$ ), and let $\varphi: C \rightarrow R$ be a real-valued function.

Recently, Ceng and Yao [6] introduced the following mixed equilibrium problem (MEP), that is, to find $z \in C$ such that

$$
\text { MEP : } \Theta(z, y)+\varphi(y)-\varphi(z) \geq 0, \quad \forall y \in C
$$


The set of solutions of (1.7) is denoted by $\operatorname{MEP}(\Theta, \varphi)$, that is,

$$
\operatorname{MEP}(\Theta)=\{z \in C: \Theta(z, y)+\varphi(y)-\varphi(z) \geq 0, \forall y \in C\}
$$

In particular, if $\varphi=0$, this problem reduces to the equilibrium problem, that is, to find $z \in C$ such that

$$
\mathrm{EP}: \Theta(z, y) \geq 0, \quad \forall y \in C
$$

Denote the set of solution of EP by $\mathrm{EP}(\Theta)$.

On the other hand, Li et al. [7] introduced two steps of iterative procedures for the approximation of common fixed point of a nonexpansive semigroup $\{T(s): 0 \leq s<\infty\}$ on a nonempty closed convex subset $C$ in a Hilbert space.

Very recently, Saeidi [8] introduced a more general iterative algorithm for finding a common element of the set of solutions for a system of equilibrium problems and of the set of common fixed points for a finite family of nonexpansive mappings and a nonexpansive semigroup.

Recall that a family of mappings $\tau=\{T(s): 0 \leq s<\infty\}: C \rightarrow C$ is called $a$ nonexpansive semigroup, if it satisfies the following conditions:

(a) $T(s+t)=T(s) T(t)$ for all $s, t \geq 0$ and $T(0)=I$;

(b) $\|T(s) x-T(s) y\| \leq\|x-y\|$, for all $x, y \in C$.

(c) the mapping $T(\cdot) x$ is continuous, for each $x \in C$.

Motivated and inspired by Ceng and Yao [6], Li et al. [7], Saeidi [8], and [9-13], the purpose of this paper is to introduce a hybrid iterative scheme for finding a common element of the set of solutions for a system of mixed equilibrium problems, the set of common fixed point for a nonexpansive semigroup, and the set of solutions of the quasi-variational inclusion problem with multivalued maximal monotone mappings and inverse-strongly monotone mappings in Hilbert space. Under suitable conditions, some strong convergence theorems are proved. Our results extend the recent results in Zhang et al. [5], S. Takahashi and W. Takahashi [14], Chang et al. [15], Ceng and Yao [6], Li et al. [7] and, Saeidi [8].

\section{Preliminaries}

In the sequel, we use $x_{n} \rightarrow x$ and $x_{n} \rightarrow x$ to denote the weak convergence and strong convergence of the sequence $\left\{x_{n}\right\}$ in $H$, respectively.

Definition 2.1. Let $M: H \rightarrow 2^{H}$ be a multivalued maximal monotone mapping, then the single-valued mapping $J_{M, \lambda}: H \rightarrow H$ defined by

$$
J_{M, \lambda}(u)=(I+\lambda M)^{-1}(u), \quad \forall u \in H
$$

is called the resolvent operator associated with $M$, where $\lambda$ is any positive number, and $I$ is the identity mapping. 
Proposition 2.2 (see [5]). (a) The resolvent operator $J_{M, \lambda}$ associated with $M$ is single-valued and nonexpansive for all $\lambda>0$, that is,

$$
\left\|J_{M, \lambda}(x)-J_{M, \lambda}(y)\right\| \leq\|x-y\|, \quad \forall x, y \in H, \forall \lambda>0 .
$$

(b) The resolvent operator $J_{M, \lambda}$ is 1-inverse-strongly monotone, that is,

$$
\left\|J_{M, \lambda}(x)-J_{M, \lambda}(y)\right\|^{2} \leq\left\langle x-y, J_{M, \lambda}(x)-J_{M, \lambda}(y)\right\rangle, \quad \forall x, y \in H .
$$

Definition 2.3. A single-valued mapping $P: H \rightarrow H$ is said to be hemicontinuous, if for any $x, y \in H$, the mapping $t \mapsto P(x+t y)$ converges weakly to $P x$ (as $t \rightarrow 0+$ ).

It is well known that every continuous mapping must be hemicontinuous.

Lemma 2.4 (see [16]). Let $E$ be a real Banach space, $E^{*}$ the dual space of $E, T: E \rightarrow 2^{E^{*}}$ a maximal monotone mapping, and $P: E \rightarrow E^{*}$ a hemicontinuous bounded monotone mapping with $D(P)=E$, then the mapping $S=T+P: E \rightarrow 2^{E^{*}}$ is a maximal monotone mapping.

For solving the equilibrium problem for bifunction $\Theta: C \times C \rightarrow R$, let us assume that $\Theta$ satisfies the following conditions:

$\left(\mathrm{H}_{1}\right) \Theta(x, x)=0$ for all $x \in C$;

$\left(\mathrm{H}_{2}\right) \Theta$ is monotone, that is, $\Theta(x, y)+\Theta(y, x) \leq 0$ for all $x, y \in C$;

$\left(\mathrm{H}_{3}\right)$ for each $y \in C, x \mapsto \Theta(x, y)$ is concave and upper semicontinuous.

$\left(\mathrm{H}_{4}\right)$ for each $x \in C, y \mapsto \Theta(x, y)$ is convex. such that

A map $\eta: C \times C \rightarrow H$ is called Lipschitz continuous, if there exists a constant $L>0$

$$
\|\eta(x, y)\| \leq L\|x-y\|, \quad \forall x, y \in C .
$$

A differentiable function $K: C \rightarrow R$ on a convex set $C$ is called

(i) $\eta$-convex $[6]$ if

$$
K(y)-K(x) \geq\left\langle K^{\prime}(x), \eta(y, x)\right\rangle, \quad \forall x, y \in C,
$$

where $\left.K^{\prime}(x)\right)$ is the Fréchet derivative of $K$ at $x$;

(ii) $\eta$-strongly convex [6] if there exists a constant $\mu>0$ such that

$$
K(y)-K(x)-\left\langle K^{\prime}(x), \eta(y, x)\right\rangle \geq\left(\frac{\mu}{2}\right)\|x-y\|^{2}, \quad \forall x, y \in C .
$$

Let $\Theta: C \times C \rightarrow R$ be an equilibrium bifunction satisfying the conditions $\left(\mathrm{H}_{1}\right)-\left(\mathrm{H}_{4}\right)$. Let $r$ be any given positive number. For a given point $x \in C$, consider the following auxiliary problem for MEP (for short, $\operatorname{MEP}(x, r)$ ) to find $y \in C$ such that

$$
\Theta(y, z)+\varphi(z)-\varphi(y)+\frac{1}{r}\left\langle K^{\prime}(y)-K^{\prime}(x), \eta(z, y)\right\rangle \geq 0, \quad \forall z \in C,
$$


where $\eta: C \times C \rightarrow H$ is a mapping, and $K^{\prime}(x)$ is the Fréchet derivative of a functional $K: C \rightarrow R$ at $x$. Let $V_{r}^{\Theta}: C \rightarrow C$ be the mapping such that for each $x \in C, V_{r}^{\Theta}(x)$ is the set of solutions of $\operatorname{MEP}(x, r)$, that is,

$$
\begin{aligned}
V_{r}^{\Theta}(x)=\{y \in C: \Theta(y, z)+\varphi(z)-\varphi(y) \\
\left.\quad+\frac{1}{r}\left\langle K^{\prime}(y)-K^{\prime}(x), \eta(z, y)\right\rangle \geq 0, \forall z \in C\right\}, \quad \forall x \in C .
\end{aligned}
$$

Then the following conclusion holds.

Proposition 2.5 (see [6]). Let $C$ be a nonempty closed convex subset of $H, \varphi: C \rightarrow R$ a lower semicontinuous and convex functional. Let $\Theta: C \times C \rightarrow R$ be an equilibrium bifunction satisfying conditions $\left(H_{1}\right)-\left(H_{4}\right)$. Assume that

(i) $\eta: C \times C \rightarrow H$ is Lipschitz continuous with constant $L>0$ such that

(a) $\eta(x, y)+\eta(y, x)=0$, for all $x, y \in C$,

(b) $\eta(\cdot, \cdot)$ is affine in the first variable,

(c) for each fixed $y \in C, x \mapsto \eta(y, x)$ is continuous from the weak topology to the weak topology;

(ii) $K: C \rightarrow R$ is $\eta$-strongly convex with constant $\mu>0$, and its derivative $K^{\prime}$ is continuous from the weak topology to the strong topology;

(iii) for each $x \in C$, there exists a bounded subset $D_{x} \subseteq C$ and $z_{x} \in C$ such that for any $y \in C \backslash D_{x}$, one has

$$
\Theta\left(y, z_{x}\right)+\varphi\left(z_{x}\right)-\varphi(y)+\frac{1}{r}\left\langle K^{\prime}(y)-K^{\prime}(x), \eta\left(z_{x}, y\right)\right\rangle<0 .
$$

Then the following hold:

(i) $V_{r}^{\Theta}$ is single-valued;

(ii) $V_{r}^{\Theta}$ is nonexpansive if $K^{\prime}$ is Lipschitz continuous with constant $v>0$ such that $\mu \geq L v$;

(iii) $F\left(V_{r}^{\Theta}\right)=\operatorname{MEP}(\Theta)$;

(iv) $\operatorname{MEP}(\Theta)$ is closed and convex.

Lemma 2.6 (see [17]). Let $C$ be a nonempty bounded closed convex subset of $H$, and let $\mathfrak{I}=\{T(s)$ : $0 \leq s<\infty$ \} be a nonexpansive semigroup on $C$, then for any $h \geq 0$

$$
\lim _{t \rightarrow \infty} \sup _{x \in C}\left\|\frac{1}{t} \int_{0}^{t} T(s) x d s-T(h)\left(\frac{1}{t} \int_{0}^{t} T(s) x d s\right)\right\|=0 .
$$


Lemma 2.7 (see [7]). Let $C$ be a nonempty bounded closed convex subset of $H$, and let $\mathfrak{I}=\{T(s)$ : $0 \leq s<\infty\}$ be a nonexpansive semigroup on $C$. If $\left\{x_{n}\right\}$ is a sequence in $C$ such that $x_{n} \rightarrow z$ and $\lim \sup _{s \rightarrow \infty} \lim \sup _{n \rightarrow \infty}\left\|T(s) x_{n}-x_{n}\right\|=0$, then $z \in F(\mathfrak{I})$.

\section{The Main Results}

In order to prove the main result, we first give the following lemma.

Lemma 3.1 (see [5]). (a) $u \in H$ is a solution of variational inclusion (1.2) if and only if $u=$ $J_{M, \lambda}(u-\lambda B u)$, for all $\lambda>0$, that is,

$$
V I(H, B, M)=F\left(J_{M, \lambda}(I-\lambda B)\right), \quad \forall \lambda>0 .
$$

(b) If $\lambda \in(0,2 \alpha]$, then $\operatorname{VI}(H, B, M)$ is a closed convex subset in $H$.

In the sequel, we assume that $H, C, M, A, B, f, T, F, \varphi_{i}, \eta_{i}, K_{i}(i=1,2, \ldots, N)$ satisfy the following conditions:

(1) $H$ is a real Hilbert space, $C \subset H$ is a nonempty closed convex subset;

(2) $A: H \rightarrow H$ is a strongly positive linear bounded operator with a coefficient $\bar{\gamma}>$ $0, f: H \rightarrow H$ is a contraction mapping with a contraction constant $h(0<h<1)$, $0<\gamma<\bar{\gamma} / h, B: C \rightarrow H$ is an $\alpha$-inverse-strongly monotone mapping, and $M$ : $H \rightarrow 2^{H}$ is a multivalued maximal monotone mapping;

(3) $\tau=\{T(s): 0 \leq s<\infty\}: C \rightarrow C$ is a nonexpansive semigroup;

(4) $\mathcal{F}=\left\{\Theta_{i}: i=1,2, \ldots, N\right\}: C \times C \rightarrow R$ is a finite family of bifunctions satisfying conditions $\left(\mathrm{H}_{1}\right)-\left(\mathrm{H}_{4}\right)$, and $\varphi_{i}: C \rightarrow R(i=1,2, \ldots, N)$ is a finite family of lower semicontinuous and convex functional;

(5) $\eta_{i}: C \times C \rightarrow H$ is a finite family of Lipschitz continuous mappings with constant $L_{i}>0(i=1,2, \ldots, N)$ such that

(a) $\eta_{i}(x, y)+\eta_{i}(y, x)=0$, for all $x, y \in C$,

(b) $\eta_{i}(\cdot, \cdot)$ is affine in the first variable,

(c) for each fixed $y \in C, x \mapsto \eta_{i}(y, x)$ is sequentially continuous from the weak topology to the weak topology;

(6) $K_{i}: C \rightarrow R$ is a finite family of $\eta_{i}$-strongly convex with constant $\mu_{i}>0$, and its derivative $K_{i}^{\prime}$ is not only continuous from the weak topology to the strong topology but also Lipschitz continuous with constant $v_{i}>0, \mu_{i} \geq L_{i} v_{i}$. 
In the sequel we always denote by $F(\tau)$ the set of fixed points of the nonexpansive semi-group $\tau, \operatorname{VI}(H, B, M)$ the set of solutions to the variational inequality (1.2), and $\operatorname{MEP}(\mathcal{F})$ the set of solutions to the following auxiliary problem for a system of mixed equilibrium problems:

$$
\begin{gathered}
\Theta_{1}\left(y_{n}^{(1)}, x\right)+\phi_{1}(x)-\phi_{1}\left(y_{n}^{(1)}\right)+\frac{1}{r_{1}}\left\langle K^{\prime}\left(y_{n}^{(1)}\right)-K^{\prime}\left(x_{n}\right), \eta_{1}\left(x, y_{n}^{(1)}\right)\right\rangle \geq 0, \quad \forall x \in C, \\
\Theta_{2}\left(y_{n}^{(2)}, x\right)+\phi_{2}(x)-\phi_{2}\left(y_{n}^{(2)}\right)+\frac{1}{r_{2}}\left\langle K^{\prime}\left(y_{n}^{(2)}\right)-K^{\prime}\left(y_{n}^{(1)}\right), \eta_{2}\left(x, y_{n}^{(2)}\right)\right\rangle \geq 0, \quad \forall x \in C, \\
\vdots \\
\Theta_{N-1}\left(y_{n}^{(N-1)}, x\right)+\phi_{N-1}(x)-\phi_{N-1}\left(y_{n}^{(N-1)}\right) \\
\quad+\frac{1}{r_{N-1}}\left\langle K^{\prime}\left(y_{n}^{(N-1)}\right)-K^{\prime}\left(y_{n}^{(N-2)}\right), \eta_{N-1}\left(x, y_{n}^{(N-1)}\right)\right\rangle \geq 0, \quad \forall x \in C, \\
\Theta_{N}\left(y_{n}, x\right)+\phi_{N}(x)-\phi_{N}\left(y_{n}\right)+\frac{1}{r_{N}}\left\langle K^{\prime}\left(y_{n}\right)-K^{\prime}\left(y_{n}^{(N-1)}\right), \eta_{N}\left(x, y_{n}\right)\right\rangle \geq 0, \quad \forall x \in C,
\end{gathered}
$$

where

$$
\begin{aligned}
y_{n}^{(1)} & =V_{r_{1}}^{\Theta_{1}} x_{n} \\
y_{n}^{(i)} & =V_{r_{i}}^{\Theta_{i}} y_{n}^{(i-1)}=V_{r_{i}}^{\Theta_{i}} V_{r_{(i-1)}}^{\Theta_{i-1}} y_{n}^{(i-2)}=V_{r_{i}}^{\Theta_{i}} \cdots V_{r_{2}}^{\Theta_{2}} y_{n}^{(1)} \\
& =V_{r_{i}}^{\Theta_{i}} \cdots V_{r_{2}}^{\Theta_{2}} V_{r_{1}}^{\Theta_{1}} x_{n}, \quad i=2,3, \ldots, N-1, \\
y_{n} & =V_{r_{N}}^{\Theta_{N}} \cdots V_{r_{2}}^{\Theta_{2}} V_{r_{1}}^{\Theta_{1}} x_{n},
\end{aligned}
$$

and $V_{r_{i}}^{\Theta_{i}}: C \rightarrow C, i=1,2, \ldots, N$ is the mapping defined by (2.8).

In the sequel we denote by $\mho^{l}=V_{r_{l}}^{\Theta_{l}} \ldots V_{r_{2}}^{\Theta_{2}} V_{r_{1}}^{\Theta_{1}}$ for $l \in\{1,2, \ldots, N\}$ and $\mho^{0}=I$.

Theorem 3.2. Let $H, C, A, B, M, f, T, F, \varphi_{i}, \eta_{i}, K_{i}(i=1,2, \ldots, N)$ be the same as above. Let $r_{i}(i=$ $1,2, \ldots, N)$ be a finite family of positive numbers, $\lambda \in(0,2 \alpha],\left\{\alpha_{n}\right\},\left\{\beta_{n}\right\} \subset[0,1]$, and $\left\{t_{n}\right\} \subset$ $(0, \infty)$. If $\mathcal{G}:=F(\tau) \cap M E P(\Psi) \cap V I(H, B, M) \neq \emptyset$ and the following conditions are satisfied:

(i) for each $x \in C$, there exists a bounded subset $D_{x} \subseteq C$ and $z_{x} \in C$ such that for any $y \in C \backslash D_{x}$

$$
\Theta_{i}\left(y, z_{x}\right)+\varphi_{i}\left(z_{x}\right)-\varphi_{i}(y)+\frac{1}{r_{i}}\left\langle K_{i}^{\prime}(y)-K_{i}^{\prime}(x), \eta_{i}\left(z_{x}, y\right)\right\rangle<0,
$$

(ii) $\lim _{n \rightarrow \infty} \alpha_{n}=0, \sum_{n=1}^{\infty} \alpha_{n}=\infty, 0<\lim _{\inf }{ }_{n \rightarrow \infty} \beta_{n} \leq \lim \sup _{n \rightarrow \infty} \beta_{n}<1$, and $\lim _{n \rightarrow \infty} t_{n}=\infty$, then 
(1) for each $n \geq 1$, there is a unique $x_{n} \in C$ such that

$$
\begin{aligned}
x_{n}= & \alpha_{n} \gamma f\left(\frac{1}{t_{n}} \int_{0}^{t_{n}} T(s) x_{n} d s\right)+ \\
& \beta_{n} x_{n}+\left(\left(1-\beta_{n}\right) I-\alpha_{n} A\right) \frac{1}{t_{n}} \int_{0}^{t_{n}} T(s)\left(J_{M, \lambda}(I-\lambda B)\right)^{2} \mho^{N} x_{n} d s,
\end{aligned}
$$

(2) the sequence $\left\{x_{n}\right\}$ converges strongly to some point $x^{*} \in \mathcal{G}$, provided that $V_{r_{i}}^{\Theta_{i}}$ is firmly nonexpansive;

(3) $x^{*}$ is the unique solution of the following variational inequality

$$
\left\langle(A-r f) x^{*}, x^{*}-z\right\rangle \leq 0, \quad \forall z \in \mathcal{G} \text {. }
$$

Proof. We observe that from condition (ii), we can assume, without loss of generality, that $\alpha_{n} \leq\left(1-\beta_{n}\right)\|A\|^{-1}$.

Since $A$ is a linear bounded self-adjoint operator on $H$, then

$$
\|A\|=\sup \{|\langle A u, u\rangle|: u \in H,\|u\|=1\} .
$$

Since

$$
\begin{aligned}
\left\langle\left(\left(1-\beta_{n}\right) I-\alpha_{n} A\right) u, u\right\rangle & =1-\beta_{n}-\alpha_{n}\langle A u, u\rangle \\
& \geq 1-\beta_{n}-\alpha_{n}\|A\| \geq 0
\end{aligned}
$$

this implies that $\left(1-\beta_{n}\right) I-\alpha_{n} A$ is positive. Hence we have

$$
\begin{aligned}
\left\|\left(1-\beta_{n}\right) I-\alpha_{n} A\right\| & =\sup \left\{\left|\left\langle\left(\left(1-\beta_{n}\right) I-\alpha_{n} A\right) u, u\right\rangle\right|: u \in H,\|u\|=1\right\} \\
& =\sup \left\{1-\beta_{n}-\alpha_{n}\langle A u, u\rangle: u \in H,\|u\|=1\right\} \\
& \leq 1-\beta_{n}-\alpha_{n} \bar{\gamma}<1 .
\end{aligned}
$$

For each given $n \geq 1$, let us define the mapping

$$
W_{n}:=\alpha_{n} \gamma f \frac{1}{t_{n}} \int_{0}^{t_{n}} T(s) d s+\beta_{n} I+\left(\left(1-\beta_{n}\right) I-\alpha_{n} A\right) \frac{1}{t_{n}} \int_{0}^{t_{n}} T(s)\left(J_{M, \lambda}(I-\lambda B)\right)^{2} \mho^{N} d s
$$


Firstly we show that the mapping $W_{n}: C \rightarrow C$ is a contraction. Indeed, for any $x, y \in C$, we have

$$
\begin{aligned}
\| W_{n} x- & W_{n} y \| \\
= & \| \alpha_{n} \gamma f\left(\frac{1}{t_{n}} \int_{0}^{t_{n}} T(s) x d s\right)+\beta_{n} x+\left(\left(1-\beta_{n}\right) I-\alpha_{n} A\right) \frac{1}{t_{n}} \int_{0}^{t_{n}} T(s)\left(J_{M, \lambda}(I-\lambda B)\right)^{2} \mho^{N} x d s \\
& \quad-\alpha_{n} \gamma f \frac{1}{t_{n}} \int_{0}^{t_{n}} T(s) y d s-\beta_{n} y-\left(\left(1-\beta_{n}\right) I-\alpha_{n} A\right) \frac{1}{t_{n}} \int_{0}^{t_{n}} T(s)\left(J_{M, \lambda}(I-\lambda B)\right)^{2} \mho^{N} y d s \| \\
\leq & \alpha_{n} \gamma\left\|f\left(\frac{1}{t_{n}} \int_{0}^{t_{n}} T(s) x d s\right)-f\left(\frac{1}{t_{n}} \int_{0}^{t_{n}} T(s) y d s\right)\right\|+\beta_{n}\|x-y\| \\
& +\left(1-\beta_{n}-\alpha_{n} \bar{\gamma}\right) \frac{1}{t_{n}} \int_{0}^{t_{n}}\left\|T(s)\left(J_{M, \lambda}(I-\lambda B)\right)^{2} \mho^{N} x-T(s)\left(J_{M, \lambda}(I-\lambda B)\right)^{2} \mho^{N} y\right\| d s \\
\leq & \alpha_{n} \gamma h\|x-y\|+\beta_{n}\|x-y\|+\left\|\left(1-\beta_{n}-\alpha_{n} \bar{\gamma}\right)\right\| x-y \| \\
= & \left(1-\alpha_{n}(\bar{\gamma}-\gamma h)\right)\|x-y\| .
\end{aligned}
$$

This implies that $W_{n}: C \rightarrow C$ is a contraction mapping. Let $x_{n} \in C$ be the unique fixed point of $W_{n}$. Thus,

$$
\begin{aligned}
x_{n}= & \alpha_{n} \gamma f\left(\frac{1}{t_{n}} \int_{0}^{t_{n}} T(s) x_{n} d s\right)+\beta_{n} x_{n} \\
& +\left(\left(1-\beta_{n}\right) I-\alpha_{n} A\right)\left(\frac{1}{t_{n}} \int_{0}^{t_{n}} T(s)\left(J_{M, \lambda}(I-\lambda B)\right)^{2} \mho^{N} x_{n} d s\right)
\end{aligned}
$$

is well defined.

Letting $y_{n}=\mho^{N} x_{n}, \xi_{n}=J_{M, \lambda}(I-\lambda B) y_{n}$, and $\rho_{n}=J_{M, \lambda}(I-\lambda B) \xi_{n}$, then

$$
x_{n}=\alpha_{n} \gamma f\left(\frac{1}{t_{n}} \int_{0}^{t_{n}} T(s) x_{n} d s\right)+\beta_{n} x_{n}+\left(\left(1-\beta_{n}\right) I-\alpha_{n} A\right) \frac{1}{t_{n}} \int_{0}^{t_{n}} T(s) \rho_{n} d s .
$$

We divide the proof of Theorem 3.2 into 8 steps.

Step 1. First prove that the sequences $\left\{x_{n}\right\},\left\{\rho_{n}\right\},\left\{\xi_{n}\right\}$, and $\left\{y_{n}\right\}$ are bounded.

(a) Pick $p \in G$, since $y_{n}=\mho^{N} x_{n}$ and $p=\mho^{N} p$, we have

$$
\left\|y_{n}-p\right\|=\left\|\mho^{N} x_{n}-p\right\| \leq\left\|x_{n}-p\right\|
$$


(b) Since $p \in \operatorname{VI}(H, B, M)$ and $\rho_{n}=J_{M, \lambda}(I-\lambda B) \xi_{n}$, we have $p=J_{M, \lambda}(I-\lambda B) p$, and so

$$
\begin{aligned}
\left\|\rho_{n}-p\right\| & =\left\|J_{M, \lambda}(I-\lambda B) \xi_{n}-J_{M, \lambda}(I-\lambda B) p\right\| \\
& \leq\left\|(I-\lambda B) \xi_{n}-(I-\lambda B) p\right\| \leq\left\|\xi_{n}-p\right\| \\
& =\left\|J_{M, \lambda}(I-\lambda B) y_{n}-J_{M, \lambda}(I-\lambda B) p\right\| \\
& \leq\left\|y_{n}-p\right\| \leq\left\|x_{n}-p\right\| .
\end{aligned}
$$

Letting $u_{n}=\left(1 / t_{n}\right) \int_{0}^{t_{n}} T(s) x_{n} d s, q_{n}=\left(1 / t_{n}\right) \int_{0}^{t_{n}} T(s) \rho_{n} d s$, we have

$$
\begin{aligned}
\left\|u_{n}-p\right\| & =\left\|\frac{1}{t_{n}} \int_{0}^{t_{n}} T(s) x_{n} d s-p\right\| \\
& \leq \frac{1}{t_{n}} \int_{0}^{t_{n}}\left\|T(s) x_{n}-T(s) p\right\| d s \\
& \leq\left\|x_{n}-p\right\| .
\end{aligned}
$$

Similarly, we have

$$
\left\|q_{n}-p\right\| \leq\left\|\rho_{n}-p\right\|
$$

Form (3.5), (3.9), (3.14), (3.15), (3.16), and (3.17) we have

$$
\begin{aligned}
\| x_{n}- & p \| \\
& =\left\|\alpha_{n} \gamma f\left(u_{n}\right)+\beta_{n} x_{n}+\left(\left(1-\beta_{n}\right) I-\alpha_{n} A\right) q_{n}-p\right\| \\
& =\left\|\alpha_{n} \gamma\left(f\left(u_{n}\right)-f(p)\right)+\beta_{n}\left(x_{n}-p\right)+\left(\left(1-\beta_{n}\right) I-\alpha_{n} A\right)\left(q_{n}-p\right)+\alpha_{n}(\gamma f(p)-A p)\right\| \\
& \leq \alpha_{n} \gamma h\left\|u_{n}-p\right\|+\beta_{n}\left\|x_{n}-p\right\|+\left(\left(1-\beta_{n}\right)-\alpha_{n} \bar{\gamma}\right)\left\|q_{n}-p\right\|+\alpha_{n}\|\gamma f(p)-A p\| \\
& \leq \alpha_{n} \gamma h\left\|x_{n}-p\right\|+\beta_{n}\left\|x_{n}-p\right\|+\left(\left(1-\beta_{n}\right)-\alpha_{n} \bar{\gamma}\right)\left\|x_{n}-p\right\|+\alpha_{n}\|\gamma f(p)-A p\| .
\end{aligned}
$$

So, $\left\|x_{n}-p\right\| \leq(1 /(\bar{\gamma}-\gamma h))\|\gamma f(p)-A p\|$. This implies that $\left\{x_{n}\right\}$ is a bounded sequence in $H$. Therefore $\left\{y_{n}\right\},\left\{\rho_{n}\right\},\left\{\xi_{n}\right\},\left\{\gamma f\left(u_{n}\right)\right\}$, and $\left\{q_{n}\right\}$ are all bounded.

Step 2. Next we prove that

$$
\left\|x_{n}-T(s) x_{n}\right\| \longrightarrow 0, \quad(n \longrightarrow \infty)
$$

Since $x_{n}=\alpha_{n} \gamma f\left(u_{n}\right)+\beta_{n} x_{n}+\left(\left(1-\beta_{n}\right) I-\alpha_{n} A\right) q_{n}$, then

$$
\left\|x_{n}-q_{n}\right\| \leq \alpha_{n}\left\|\gamma f\left(u_{n}\right)-A q_{n}\right\|+\beta_{n}\left\|x_{n}-q_{n}\right\| .
$$


Hence

$$
\left\|x_{n}-q_{n}\right\| \leq \frac{\alpha_{n}}{1-\beta_{n}}\left\|\gamma f\left(u_{n}\right)-A q_{n}\right\| .
$$

From condition (ii), we have

$$
\left\|x_{n}-q_{n}\right\| \longrightarrow 0
$$

Let $K=\{w \in C:\|w-p\| \leq(1 /(\bar{\gamma}-\gamma h))\|\gamma f(p)-A p\|\}$, then $K$ is a nonempty bounded closed convex subset of $C$ and $T(s)$-invariant. Since $\left\{x_{n}\right\} \subset K$ and $K$ is bounded, there exists $r>0$ such that $K \subset B_{r}$; it follows from Lemma 2.6 that

$$
\lim _{n \rightarrow \infty}\left\|q_{n}-T(s) q_{n}\right\| \longrightarrow 0 .
$$

From (3.22) and (3.23), we have

$$
\begin{aligned}
\left\|x_{n}-T(s) x_{n}\right\| & =\left\|x_{n}-q_{n}+q_{n}-T(s) q_{n}+T(s) q_{n}-T(s) x_{n}\right\| \\
& \leq\left\|x_{n}-q_{n}\right\|+\left\|q_{n}-T(s) q_{n}\right\|+\left\|T(s) q_{n}-T(s) x_{n}\right\| \\
& \leq\left\|x_{n}-q_{n}\right\|+\left\|q_{n}-T(s) q_{n}\right\|+\left\|q_{n}-x_{n}\right\| \longrightarrow 0 .
\end{aligned}
$$

Step 3. Next we prove that

$$
\text { (i) } \lim _{n \rightarrow \infty}\left\|\mho^{l+1} x_{n}-\mho^{l} x_{n}\right\|=0, \quad \forall l \in\{0,1, \ldots, N-1\} \text {; }
$$

(ii) especially, $\lim _{n \rightarrow \infty}\left\|\mho^{N} x_{n}-x_{n}\right\|=\lim _{n \rightarrow \infty}\left\|y_{n}-x_{n}\right\|=0$.

In fact, for any given $p \in G$ and $l \in\{0,1, \ldots, N-1\}$, since $V_{r_{l+1}}^{\Theta_{l+1}}$ is firmly nonexpansive, we have

$$
\begin{aligned}
\left\|\mho^{l+1} x_{n}-p\right\|^{2} & =\left\|V_{r_{l+1}}^{\Theta_{l+1}}\left(\mho^{l} x_{n}\right)-V_{r_{l+1}}^{\Theta_{l+1}} p\right\|^{2} \\
& \leq\left\langle V_{r_{l+1}}^{\Theta_{l+1}}\left(\mho^{l} x_{n}\right)-p, \mho^{l} x_{n}-p\right\rangle \\
& =\left\langle\mho^{l+1} x_{n}-p, \mho^{l} x_{n}-p\right\rangle \\
& =\frac{1}{2}\left(\left\|\mho^{l+1} x_{n}-p\right\|^{2}+\left\|\mho^{l} x_{n}-p\right\|^{2}-\left\|\mho^{l} x_{n}-\mho^{l+1} x_{n}^{\prime}\right\|^{2}\right) .
\end{aligned}
$$

It follows that

$$
\left\|\mho^{l+1} x_{n}-p\right\|^{2} \leq\left\|x_{n}-p\right\|^{2}-\left\|\mho^{l} x_{n}-\mho^{l+1} x_{n}\right\|^{2}
$$


From (3.5), we have

$$
\begin{aligned}
\left\|x_{n}-p\right\|^{2}= & \left\|\alpha_{n} \gamma f\left(u_{n}\right)+\beta_{n} x_{n}+\left(\left(1-\beta_{n}\right) I-\alpha_{n} A\right) q_{n}-p\right\|^{2} \\
= & \left\|\alpha_{n}\left(\gamma f\left(u_{n}\right)-A p\right)+\beta_{n}\left(x_{n}-q_{n}\right)+\left(I-\alpha_{n} A\right)\left(q_{n}-p\right)\right\|^{2} \\
\leq & \left\|\left(I-\alpha_{n} A\right)\left(q_{n}-p\right)+\beta_{n}\left(x_{n}-q_{n}\right)\right\|^{2}+2 \alpha_{n}\left\langle\gamma f\left(u_{n}\right)-A p, x_{n}-p\right\rangle \\
\leq & {\left[\left\|\left(I-\alpha_{n} A\right)\left(q_{n}-p\right)\right\|+\beta_{n}\left\|\left(x_{n}-q_{n}\right)\right\|\right]^{2}+2 \alpha_{n}\left\langle\gamma f\left(u_{n}\right)-A p, x_{n}-p\right\rangle } \\
\leq & {\left[\left(1-\alpha_{n} \bar{\gamma}\right)\left\|\rho_{n}-p\right\|+\beta_{n}\left\|x_{n}-q_{n}\right\|\right]^{2}+2 \alpha_{n}\left\langle\gamma f\left(u_{n}\right)-A p, x_{n}-p\right\rangle } \\
= & \left(1-\alpha_{n} \bar{\gamma}\right)^{2}\left\|\rho_{n}-p\right\|^{2}+\beta_{n}^{2}\left\|x_{n}-q_{n}\right\|^{2}+2\left(1-\alpha_{n} \bar{\gamma}\right) \beta_{n}\left\|\rho_{n}-p\right\| \cdot\left\|x_{n}-q_{n}\right\| \\
& +2 \alpha_{n}\left\|\gamma f\left(u_{n}\right)-A p\right\| \cdot\left\|x_{n}-p\right\| .
\end{aligned}
$$

Since

$$
\left\|\rho_{n}-p\right\| \leq\left\|\xi_{n}-p\right\| \leq\left\|\mho^{N} x_{n}-p\right\| \leq\left\|\mho^{l+1} x_{n}-p\right\|, \quad \forall l \in\{0,1, \ldots, N-1\},
$$

and this together with (3.27) and (3.28), it yields

$$
\begin{aligned}
\| x_{n}- & p \|^{2} \\
\leq & \left(1-\alpha_{n} \bar{\gamma}\right)^{2}\left\{\left\|x_{n}-p\right\|^{2}-\left\|\mho^{l} x_{n}-\mho^{l+1} x_{n}\right\|^{2}\right\}+\beta_{n}^{2}\left\|x_{n}-q_{n}\right\|^{2} \\
& +2\left(1-\alpha_{n} \bar{\gamma}\right) \cdot \beta_{n}\left\|\rho_{n}-p\right\| \cdot\left\|x_{n}-q_{n}\right\|+2 \alpha_{n}\left\|\gamma f\left(u_{n}\right)-A p\right\| \cdot\left\|x_{n}-p\right\| \\
= & \left(1-2 \alpha_{n} \bar{\gamma}+\left(\alpha_{n} \bar{\gamma}\right)^{2}\right)\left\|x_{n}-p\right\|^{2}-\left(1-\alpha_{n} \bar{\gamma}\right)^{2}\left\|\mho^{l} x_{n}-\mho^{l+1} x_{n}\right\|^{2}+\beta_{n}^{2}\left\|x_{n}-q_{n}\right\|^{2} \\
& +2\left(1-\alpha_{n} \bar{\gamma}\right) \beta_{n}\left\|\rho_{n}-p\right\| \cdot\left\|x_{n}-q_{n}\right\|+2 \alpha_{n}\left\|\gamma f\left(u_{n}\right)-A p\right\| \cdot\left\|x_{n}-p\right\| .
\end{aligned}
$$

Simplifying it we have

$$
\begin{aligned}
\left(1-\alpha_{n} \bar{\gamma}\right)^{2}\left\|\mho^{l} x_{n}-\mho^{l+1} x_{n}\right\|^{2} \leq & \left(1+\alpha_{n}(\bar{\gamma})^{2}\right)\left\|x_{n}-p\right\|^{2}-\left\|x_{n}-p\right\|^{2} \\
& +\beta_{n}^{2}\left\|x_{n}-q_{n}\right\|^{2}+2\left(1-\alpha_{n} \bar{\gamma}\right) \beta_{n}\left\|\rho_{n}-p\right\| \cdot\left\|x_{n}-q_{n}\right\| \\
& +2 \alpha_{n}\left\|\gamma f\left(u_{n}\right)-A p\right\| \cdot\left\|x_{n}-p\right\| .
\end{aligned}
$$

Since $\alpha_{n} \rightarrow 0$ and $\left\|x_{n}-q_{n}\right\| \rightarrow 0$, by condition (ii), it yields $\left\|\mho^{l+1} x_{n}-\mho^{l} x_{n}\right\| \rightarrow 0$.

Step 4. Now we prove that for any given $p \in G$

$$
\lim _{n \rightarrow \infty}\left\|B y_{n}-B p\right\|=0
$$


In fact, it follows from (3.15) that

$$
\begin{aligned}
\left\|\rho_{n}-p\right\|^{2} & \leq\left\|\xi_{n}-p\right\|^{2}=\left\|J_{M, \lambda}(I-\lambda B) y_{n}-J_{M, \lambda}(I-\lambda B) p\right\|^{2} \\
& \leq\left\|(I-\lambda B) y_{n}-(I-\lambda B) p\right\|^{2} \\
& =\left\|y_{n}-p\right\|^{2}-2 \lambda\left\langle y_{n}-p, B y_{n}-B p\right\rangle+\lambda^{2}\left\|B y_{n}-B p\right\|^{2} \\
& \leq\left\|y_{n}-p\right\|^{2}+\lambda(\lambda-2 \alpha)\left\|B y_{n}-B p\right\|^{2} \\
& \leq\left\|x_{n}-p\right\|^{2}+\lambda(\lambda-2 \alpha)\left\|B y_{n}-B p\right\|^{2} .
\end{aligned}
$$

Substituting (3.33) into (3.28), we obtain

$$
\begin{aligned}
\left\|x_{n}-p\right\|^{2} \leq & \left(1-\alpha_{n} \bar{\gamma}\right)^{2}\left\{\left\|x_{n}-p\right\|^{2}+\lambda(\lambda-2 \alpha)\left\|B y_{n}-B p\right\|^{2}\right\}+\beta_{n}^{2}\left\|x_{n}-q_{n}\right\|^{2} \\
& +2\left(1-\alpha_{n} \bar{\gamma}\right) \beta_{n}\left\|\rho_{n}-p\right\| \cdot\left\|x_{n}-q_{n}\right\|+2 \alpha_{n}\left\|\gamma f\left(u_{n}\right)-A p\right\| \cdot\left\|x_{n}-p\right\| .
\end{aligned}
$$

Simplifying it, we have

$$
\begin{aligned}
&\left(1-\alpha_{n} \bar{\gamma}\right)^{2} \lambda(2 \alpha-\lambda)\left\|B y_{n}-B p\right\|^{2} \\
& \leq\left(1+\alpha_{n}(\bar{\gamma})^{2}\right)\left\|x_{n}-p\right\|^{2}-\left\|x_{n}-p\right\|^{2}+\beta_{n}^{2}\left\|x_{n}-q_{n}\right\|^{2} \\
&+2\left(1-\alpha_{n} \bar{\gamma}\right) \beta_{n}\left\|\rho_{n}-p\right\| \cdot\left\|x_{n}-q_{n}\right\|+2 \alpha_{n}\left\|\gamma f\left(u_{n}\right)-A p\right\| \cdot\left\|x_{n}-p\right\| \\
&= \alpha_{n}(\bar{\gamma})^{2}\left\|x_{n}-p\right\|^{2}+\beta_{n}^{2}\left\|x_{n}-q_{n}\right\|^{2} \\
&+2\left(1-\alpha_{n} \bar{\gamma}\right) \beta_{n}\left\|\rho_{n}-p\right\| \cdot\left\|x_{n}-q_{n}\right\|+2 \alpha_{n}\left\|\gamma f\left(u_{n}\right)-A p\right\| \cdot\left\|x_{n}-p\right\| .
\end{aligned}
$$

Since $\alpha_{n} \rightarrow 0,0<\liminf _{n \rightarrow \infty} \beta_{n} \leq \lim \sup _{n \rightarrow \infty} \beta_{n}<1,\left\|x_{n}-q_{n}\right\| \rightarrow 0$, and $\left\{\gamma f\left(u_{n}\right)-A p\right\},\left\{x_{n}\right\}$ are bounded, these imply that $\left\|B y_{n}-B p\right\| \rightarrow 0(n \rightarrow \infty)$.

Step 5. Next we prove that

$$
\begin{aligned}
& \lim _{n \rightarrow \infty}\left\|y_{n}-\rho_{n}\right\|=0, \\
& \lim _{n \rightarrow \infty}\left\|x_{n}-\rho_{n}\right\|=0 .
\end{aligned}
$$

In fact, since

$$
\left\|y_{n}-\rho_{n}\right\| \leq\left\|y_{n}-\xi_{n}\right\|+\left\|\xi_{n}-\rho_{n}\right\|
$$

for the purpose, it is sufficient to prove

$$
\left\|y_{n}-\xi_{n}\right\| \longrightarrow 0, \quad\left\|\xi_{n}-\rho_{n}\right\| \longrightarrow 0
$$


(a) First we prove that $\left\|y_{n}-\xi_{n}\right\| \rightarrow 0$. In fact, since

$$
\begin{aligned}
\| \xi_{n}- & p \|^{2} \\
& =\left\|J_{M, \lambda}(I-\lambda B) y_{n}-J_{M, \lambda}(I-\lambda B) p\right\|^{2} \\
& \leq\left\langle y_{n}-\lambda B y_{n}-(p-\lambda B p), \xi_{n}-p\right\rangle \\
& =\frac{1}{2}\left\{\left\|y_{n}-\lambda B y_{n}-(p-\lambda B p)\right\|^{2}+\left\|\xi_{n}-p\right\|^{2}-\left\|y_{n}-\lambda B y_{n}-(p-\lambda B p)-\left(\xi_{n}-p\right)\right\|^{2}\right\} \\
& \leq \frac{1}{2}\left\{\left\|y_{n}-p\right\|^{2}+\left\|\xi_{n}-p\right\|^{2}-\left\|y_{n}-\xi_{n}-\lambda\left(B y_{n}-B p\right)\right\|^{2}\right\} \\
& \leq \frac{1}{2}\left\{\left\|y_{n}-p\right\|^{2}+\left\|\xi_{n}-p\right\|^{2}-\left\|y_{n}-\xi_{n}\right\|^{2}+2 \lambda\left\langle y_{n}-\xi_{n}, B y_{n}-B p\right\rangle-\lambda^{2}\left\|B y_{n}-B p\right\|^{2}\right\},
\end{aligned}
$$

we have

$$
\left\|\xi_{n}-p\right\|^{2} \leq\left\|y_{n}-p\right\|^{2}-\left\|y_{n}-\xi_{n}\right\|^{2}+2 \lambda\left\langle y_{n}-\xi_{n}, B y_{n}-B p\right\rangle-\lambda^{2}\left\|B y_{n}-B p\right\|^{2}
$$

Substituting (3.40) into (3.28), it yields that

$$
\begin{aligned}
\left\|x_{n}-p\right\|^{2} \leq & \left(1-\alpha_{n} \bar{\gamma}\right)^{2}\left\{\left\|y_{n}-p\right\|^{2}-\left\|y_{n}-\xi_{n}\right\|^{2}+2 \lambda\left\langle y_{n}-\xi_{n}, B y_{n}-B p\right\rangle\right. \\
& \left.-\lambda^{2}\left\|B y_{n}-B p\right\|^{2}\right\}+\beta_{n}^{2}\left\|x_{n}-q_{n}\right\|^{2} \\
& +2\left(1-\alpha_{n} \bar{\gamma}\right) \beta_{n}\left\|\rho_{n}-p\right\| \cdot\left\|x_{n}-q_{n}\right\|+2 \alpha_{n}\left\|\gamma f\left(u_{n}\right)-A p\right\| \cdot\left\|x_{n}-p\right\| .
\end{aligned}
$$

Simplifying it we have

$$
\begin{aligned}
\left(1-\alpha_{n} \bar{\gamma}\right)^{2}\left\|y_{n}-\xi_{n}\right\|^{2} \leq & \alpha_{n} \bar{\gamma}^{2}\left\|x_{n}-p\right\|^{2}+2\left(1-\alpha_{n} \bar{\gamma}^{2}\right) \lambda\left\langle y_{n}-\xi_{n}, B y_{n}-B p\right\rangle \\
& -\left(1-\alpha_{n} \bar{\gamma}\right)^{2} \lambda^{2}\left\|B y_{n}-B p\right\|^{2}+\beta_{n}^{2}\left\|x_{n}-q_{n}\right\|^{2} \\
& +2\left(1-\alpha_{n} \bar{\gamma}\right) \beta_{n}\left\|\rho_{n}-p\right\| \cdot\left\|x_{n}-q_{n}\right\|+2 \alpha_{n}\left\|\gamma f\left(u_{n}\right)-A p\right\| \cdot\left\|x_{n}-p\right\| .
\end{aligned}
$$

Since $\alpha_{n} \rightarrow 0,0<\lim _{\inf _{n \rightarrow \infty}} \beta_{n} \leq \lim \sup _{n \rightarrow \infty} \beta_{n}<1,\left\|x_{n}-q_{n}\right\| \rightarrow 0,\left\|B y_{n}-B p\right\| \rightarrow 0(n \rightarrow$ $\infty)$, and $\left\{\gamma f\left(u_{n}\right)-A p\right\},\left\{x_{n}\right\},\left\{\rho_{n}\right\}$ are bounded, these imply that $\left\|y_{n}-\xi_{n}\right\| \rightarrow 0(n \rightarrow \infty)$.

(b) Next we prove that

$$
\lim _{n \rightarrow \infty}\left\|\xi_{n}-\rho_{n}\right\|=0
$$

In fact, since $\left\|\xi_{n}-\rho_{n}\right\|=\left\|J_{M, \lambda}(I-\lambda B) y_{n}-J_{M, \lambda}(I-\lambda B) \xi_{n}\right\| \leq\left\|y_{n}-\xi_{n}\right\| \rightarrow 0$, so $\left\|y_{n}-\rho_{n}\right\|=\left\|y_{n}-\xi_{n}+\xi_{n}-\rho_{n}\right\| \leq\left\|y_{n}-\xi_{n}\right\|+\left\|\xi_{n}-\rho_{n}\right\| \rightarrow 0$. This together with (3.25) shows that $\left\|x_{n}-\rho_{n}\right\| \rightarrow 0$. 
Step 6. Next we prove that there exists a subsequence $\left\{x_{n_{k}}\right\}$ of $\left\{x_{n}\right\}$ such that $x_{n_{k}} \rightarrow x^{*} \in G$, and $x^{*}$ is the unique solution of the variational inequality (3.6).

(a) We first prove that $x^{*} \in F(\tau)$. In fact, since $\left\{x_{n}\right\}$ is bounded, there exists a subsequence $\left\{x_{n_{k}}\right\}$ of $\left\{x_{n}\right\}$ such that $\left\{x_{n_{k}}\right\} \rightarrow x^{*}$. From Lemma 2.7 and Step 2, we obtain $x^{*} \in F(\tau)$.

(b) Now we prove that $x^{*} \in \cap_{l=1}^{N} \operatorname{MEP}\left(\Theta_{l}, \varphi_{l}\right)$.

Since $x_{n_{k}} \rightarrow x^{*}$ and noting Step 3, without loss of generality, we may assume that $\mho^{l} x_{n_{k}} \rightarrow x^{*}$, for all $l \in\{0,1,2, \ldots, N-1\}$. Hence for any $x \in C$ and for any $l \in\{0,1,2, \ldots, N-$ $1\}$, we have

$$
\left\langle\frac{K_{l+1}^{\prime}\left(\mho^{l+1} x_{n_{k}}\right)-K_{l+1}^{\prime}\left(\mho^{l} x_{n_{k}}\right)}{r_{l+1}}, \eta_{l+1}\left(x, \mho^{l+1} x_{n_{k}}\right)\right\rangle \geq-\Theta_{l+1}\left(\mho^{l+1} x_{n_{k}}, x\right)-\varphi_{l+1}(x)+\varphi_{l+1}\left(\mho^{l+1} x_{n_{k}}\right) .
$$

By the assumptions and by condition $\left(\mathrm{H}_{2}\right)$ we know that the function $\varphi_{i}$ and the mapping $x \mapsto\left(-\Theta_{l+1}(x, y)\right)$ both are convex and lower semicontinuous, hence they are weakly lower semicontinuous. These together with $\left(K_{l+1}^{\prime}\left(\mho^{l+1} x_{n_{k}}\right)-K_{l+1}^{\prime}\left(\mho^{l} x_{n_{k}}\right)\right) / r_{l+1} \rightarrow 0$ and $\mho^{l+1} x_{n_{k}} \rightarrow$ $x^{*}$, we have

$$
\begin{aligned}
& 0=\liminf _{k \rightarrow \infty}\left\{\left\langle\frac{K_{l+1}^{\prime}\left(\mho^{l+1} x_{n_{k}}\right)-K_{l+1}^{\prime}\left(\mho^{l} x_{n_{k}}\right)}{r_{l+1}}, \eta_{l+1}\left(x, \mho^{l+1} x_{n_{k}}\right)\right\rangle\right\} \\
& \geq \liminf _{k \rightarrow \infty}\left\{-\Theta_{l+1}\left(\mho^{l+1} x_{n_{k}}, x\right)-\varphi_{l+1}(x)+\varphi_{l+1}\left(\mho^{l+1} x_{n_{k}}\right)\right\} .
\end{aligned}
$$

That is,

$$
\Theta_{l+1}\left(x^{*}, x\right)+\varphi_{l+1}(x)-\varphi_{l+1}\left(x^{*}\right) \geq 0
$$

for all $x \in C$ and $l \in\{0,1, \ldots, N-1\}$, hence $x^{*} \in \cap_{l=1}^{N} \operatorname{MEP}\left(\Theta_{l}, \varphi_{l}\right)$.

(c) Now we prove that $x^{*} \in \operatorname{VI}(H, B, M)$.

In fact, since $B$ is $\alpha$-inverse-strongly monotone, it follows from Proposition 1.1 that $B$ is a $1 / \alpha$-Lipschitz continuous monotone mapping and $D(B)=H$ (where $D(B)$ is the domain of $B)$. It follows from Lemma 2.4 that $M+B$ is maximal monotone. Let $(v, g) \in \operatorname{Graph}(M+B)$, that is, $g-B v \in M(v)$. Since $x_{n_{k}}-x^{*}$ and noting Step 3, without loss of generality, we may assume that $\mho^{l} x_{n_{k}} \rightarrow x^{*}$; in particular, we have $y_{n_{k}}=\mho^{N} x_{n_{k}} \rightarrow x^{*}$. From $\left\|y_{n}-\rho_{n}\right\| \rightarrow 0$, we can prove that $\rho_{n_{k}} \rightarrow x^{*}$. Again since $\rho_{n_{k}}=J_{M, \lambda}(I-\lambda B) \xi_{n_{k}}$, we have

$$
\xi_{n_{k}}-\lambda B \xi_{n_{k}} \in(I+\lambda M) \rho_{n_{k}} \text {, that is, } \frac{1}{\lambda}\left(\xi_{n_{k}}-\rho_{n_{k}}-\lambda B \xi_{n_{k}}\right) \in M\left(\rho_{n_{k}}\right) .
$$

By virtue of the maximal monotonicity of $M$, we have

$$
\left\langle v-\rho_{n_{k}}, g-B v-\frac{1}{\lambda}\left(\xi_{n_{k}}-\rho_{n_{k}}-\lambda B \xi_{n_{k}}\right)\right\rangle \geq 0
$$


So,

$$
\begin{aligned}
\left\langle v-\rho_{n_{k}}, g\right\rangle & \geq\left\langle v-\rho_{n_{k}}, B v+\frac{1}{\lambda}\left(\xi_{n_{k}}-\rho_{n_{k}}-\lambda B \xi_{n_{k}}\right)\right\rangle \\
& =\left\langle v-\rho_{n_{k}}, B v-B \rho_{n_{k}}+B \rho_{n_{k}}-B \xi_{n_{k}}+\frac{1}{\lambda}\left(\xi_{n_{k}}-\rho_{n_{k}}\right)\right\rangle \\
& \geq 0+\left\langle v-\rho_{n_{k}}, B \rho_{n_{k}}-B \xi_{n_{k}}\right\rangle+\left\langle v-\rho_{n_{k}}, \frac{1}{\lambda}\left(\xi_{n_{k}}-\rho_{n_{k}}\right)\right\rangle .
\end{aligned}
$$

Since $\left\|\xi_{n}-\rho_{n}\right\| \rightarrow 0,\left\|B \xi_{n}-B \rho_{n}\right\| \rightarrow 0$, and $\rho_{n_{k}} \rightarrow x^{*}$, we have

$$
\lim _{n_{k} \rightarrow \infty}\left\langle v-\rho_{n_{k}}, g\right\rangle=\left\langle v-x^{*}, g\right\rangle \geq 0 .
$$

Since $M+B$ is maximal monotone, this implies that $\theta \in(M+B)\left(x^{*}\right)$, that is, $x^{*} \in \operatorname{VI}(H, B, M)$, and so $x^{*} \in \mathcal{G}$.

(d) Now we prove that $x^{*}$ is the unique solution of variational inequality (3.6).

$\left(1^{0}\right)$ We first prove that $\left\{x_{n_{k}}\right\} \rightarrow x^{*}$.

Since for all $z \in G$,

$$
\begin{aligned}
\left\|x_{n}-z\right\|^{2} & =\left\langle x_{n}-z, x_{n}-z\right\rangle \\
& =\left\langle\alpha_{n} \gamma f\left(u_{n}\right)+\beta_{n} x_{n}+\left(\left(1-\beta_{n}\right) I-\alpha_{n} A\right) q_{n}-z, x_{n}-z\right\rangle \\
& =\left\langle\alpha_{n}\left(\gamma f\left(u_{n}\right)-A z\right)+\beta_{n}\left(x_{n}-z\right)+\left(\left(1-\beta_{n}\right) I-\alpha_{n} A\right)\left(q_{n}-z\right), x_{n}-z\right\rangle \\
& \leq \alpha_{n}\left\langle\gamma f\left(u_{n}\right)-A z, x_{n}-z\right\rangle+\beta_{n}\left\|x_{n}-z\right\|^{2}+\left(1-\beta_{n}-\alpha_{n} \bar{\gamma}\right)\left\|q_{n}-z\right\| \cdot\left\|x_{n}-z\right\| \\
& \leq\left(1-\alpha_{n} \bar{\gamma}\right)\left\|x_{n}-z\right\|^{2}+\alpha_{n}\left\langle\gamma f\left(u_{n}\right)-A z, x_{n}-z\right\rangle .
\end{aligned}
$$

It follows that

$$
\begin{aligned}
\left\|x_{n}-z\right\|^{2} & \leq \frac{1}{\bar{\gamma}}\left\langle\gamma f\left(u_{n}\right)-A z, x_{n}-z\right\rangle \\
& =\frac{1}{\bar{\gamma}}\left\langle\gamma f\left(u_{n}\right)-\gamma f(z)+\gamma f(z)-A z, x_{n}-z\right\rangle \\
& \leq \frac{1}{\bar{\gamma}}\left\{\gamma h\left\|x_{n}-z\right\|^{2}+\left\langle\gamma f(z)-A z, x_{n}-z\right\rangle\right\} .
\end{aligned}
$$


Therefore,

$$
\left\|x_{n}-z\right\|^{2} \leq \frac{1}{\bar{\gamma}-\gamma h}\left\langle\gamma f(z)-A z, x_{n}-z\right\rangle
$$

Now, replacing $n$ in (3.53) with $n_{k}$ and letting $k \rightarrow \infty$ and $x_{n_{k}} \rightarrow x^{*}$, we have $x_{n_{k}} \rightarrow x^{*}$.

$\left(2^{0}\right)$ Next we prove that $x^{*}$ is the unique solution of the variational inequality (3.6).

Since

$$
x_{n}=\alpha_{n} \gamma f\left(\frac{1}{t_{n}} \int_{0}^{t_{n}} T(s) x_{n} d s\right)+\beta_{n} x_{n}+\left(\left(1-\beta_{n}\right) I-\alpha_{n} A\right) \frac{1}{t_{n}} \int_{0}^{t_{n}} T(s) \rho_{n} d s,
$$

we have

$$
\begin{aligned}
\alpha_{n}(A & -\gamma f)\left(\frac{1}{t_{n}} \int_{0}^{t_{n}} T(s) x_{n} d s\right) \\
& =-\left\{\left(1-\beta_{n}\right)\left(x_{n}-\frac{1}{t_{n}} \int_{0}^{t_{n}} T(s) \rho_{n} d s\right)\right\}+\alpha_{n} A \frac{1}{t_{n}} \int_{0}^{t_{n}}\left(T(s) x_{n}-T(s) \rho_{n}\right) d s \\
& =-\left(1-\beta_{n}\right)\left(I-\frac{1}{t_{n}} \int_{0}^{t_{n}} T(s)\left(J_{M, \lambda}(I-\lambda B)\right)^{2} \mho^{N} d s\right) x_{n}+\alpha_{n} A \frac{1}{t_{n}} \int_{0}^{t_{n}}\left(T(s) x_{n}-T(s) \rho_{n}\right) d s .
\end{aligned}
$$

Hence for any $z \in G$ we have,

$$
\begin{aligned}
& \alpha_{n}\left\langle(A-\gamma f)\left(\frac{1}{t_{n}} \int_{0}^{t_{n}} T(s) x_{n} d s\right), x_{n}-z\right\rangle \\
& =-\left(1-\beta_{n}\right)\left\langle\left(I-\frac{1}{t_{n}} \int_{0}^{t_{n}} T(s)\left(J_{M, \lambda}(I-\lambda B)\right)^{2} \mho^{N} d s\right) x_{n}\right. \\
& \left.-\left(I-\frac{1}{t_{n}} \int_{0}^{t_{n}} T(s)\left(J_{M, \lambda}(I-\lambda B)\right)^{2} \mho^{N} d s\right) z, x_{n}-z\right\rangle \\
& +\alpha_{n}\left\langle A \frac{1}{t_{n}} \int_{0}^{t_{n}}\left(T(s) x_{n}-T(s) \rho_{n}\right) d s, x_{n}-z\right\rangle,
\end{aligned}
$$


then

$$
\begin{aligned}
\langle(A-\gamma f) & \left.\left(\frac{1}{t_{n}} \int_{0}^{t_{n}} T(s) x_{n} d s\right), x_{n}-z\right\rangle \\
= & -\frac{1-\beta_{n}}{\alpha_{n}} \\
\times & \left\langle\left(I-\frac{1}{t_{n}} \int_{0}^{t_{n}} T(s) J_{M, \lambda}^{2}(I-\lambda B) \mho^{N} d s\right) x_{n}\right. \\
& \left.\quad-\left(I-\frac{1}{t_{n}} \int_{0}^{t_{n}} T(s) J_{M, \lambda}^{2}(I-\lambda B) \mho^{N} d s\right) z, x_{n}-z\right\rangle \\
+ & \left\langle A \frac{1}{t_{n}} \int_{0}^{t_{n}}\left(T(s) x_{n}-T(s) \rho_{n}\right) d s, x_{n}-z\right\rangle .
\end{aligned}
$$

It is easily seen that $I-\left(1 / t_{n}\right) \int_{0}^{t_{n}} T(s)\left(J_{M, \lambda}(I-\lambda B)\right)^{2} \mho^{N} d s$ is monotone. Thus from (3.57) we have that

$$
\left\langle(A-\gamma \mathrm{f})\left(\frac{1}{t_{n}} \int_{0}^{t_{n}} T(s) x_{n} d s\right), x_{n}-z\right\rangle \leq\left\langle A \frac{1}{t_{n}} \int_{0}^{t_{n}}\left(T(s) x_{n}-T(s) \rho_{n}\right) d s, x_{n}-z\right\rangle .
$$

Now, in (3.58) replacing $n$ by $n_{k}$ and letting $k \rightarrow \infty$ and $x_{n_{k}} \rightarrow x^{*}$, from (3.36), we have

$$
\frac{1}{t_{n_{k}}} \int_{0}^{t_{n_{k}}}\left(T(s) x_{n_{k}}-T(s) \rho_{n_{k}}\right) d s \longrightarrow 0
$$

So, we have

$$
\left\langle(A-\gamma f) x^{*}, x^{*}-z\right\rangle \leq 0 \quad \forall z \in \mathcal{G} \text {. }
$$

It follows from [18, Theorem 3.2] that the solution of the variational inequality (3.6) is unique, that is, $x^{*}$ is a unique solution of (3.6).

Step 7. Next we prove that

$$
\limsup _{n \rightarrow \infty}\left\langle\gamma f\left(x^{*}\right)-A x^{*}, x_{n}-x^{*}\right\rangle \leq 0 .
$$

(a) First, we prove that

$$
\limsup _{n \rightarrow \infty}\left\langle\frac{1}{t_{n}} \int_{0}^{t_{n}} T(s) \rho_{n} d s-x^{*}, \gamma f\left(x^{*}\right)-A x^{*}\right\rangle \leq 0 .
$$


Indeed, there exists a subsequence $\left\{\rho_{n_{i}}\right\}$ of $\left\{\rho_{n}\right\}$ such that

$$
\limsup _{n \rightarrow \infty}\left\langle\frac{1}{t_{n}} \int_{0}^{t_{n}} T(s) \rho_{n} d s-x^{*}, \gamma f\left(x^{*}\right)-A x^{*}\right\rangle=\lim _{i \rightarrow \infty}\left\langle\frac{1}{t_{n_{i}}} \int_{0}^{t_{n_{i}}} T(s) \rho_{n_{i}} d s-x^{*}, \gamma f\left(x^{*}\right)-A x^{*}\right\rangle .
$$

We may also assume that $\rho_{n_{i}}-w$. This together with (3.22) and (3.36) shows that $q_{n_{i}}=$ $\left(1 / t_{n_{i}}\right) \int_{0}^{t_{n_{i}}} T(s) \rho_{n_{i}} d s \rightarrow w$. Since $\left\|x_{n}-q_{n}\right\| \rightarrow 0$, we have $x_{n_{i}}-w$. Again by the same method as given in Step 6 we can prove that $w \in \mathcal{G}$. So, we have

$$
\begin{aligned}
\limsup _{n \rightarrow \infty}\left\langle\frac{1}{t_{n}} \int_{0}^{t_{n}} T(s) \rho_{n} d s-x^{*}, \gamma f\left(x^{*}\right)-A x^{*}\right\rangle \\
=\lim _{i \rightarrow \infty}\left\langle\frac{1}{t_{n_{i}}} \int_{0}^{t_{n_{i}}} T(s) \rho_{n_{i}} d s-x^{*}, \gamma f\left(x^{*}\right)-A x^{* \prime}\right\rangle \\
=\lim _{i \rightarrow \infty}\left\langle q_{n_{i}}-x^{*}, \gamma f\left(x^{*}\right)-A x^{*}\right\rangle \\
=\left\langle w-x^{*}, \gamma f\left(x^{*}\right)-A x^{*}\right\rangle \leq 0 .
\end{aligned}
$$

(b) Now we prove that

$$
\limsup _{n \rightarrow \infty}\left\langle\gamma f\left(x^{*}\right)-A x^{*}, x_{n}-x^{*}\right\rangle \leq 0 .
$$

From $\left\|x_{n}-q_{n}\right\| \rightarrow 0$ and (a), we have

$$
\begin{aligned}
& \limsup _{n \rightarrow \infty}\left\langle\gamma f\left(x^{*}\right)-A x^{*}, x_{n}-x^{*}\right\rangle \\
& \quad=\limsup _{n \rightarrow \infty}\left\langle\gamma f\left(x^{*}\right)-A x^{*}, x_{n}-q_{n}+q_{n}-x^{*}\right\rangle \\
& \quad \leq \limsup _{n \rightarrow \infty}\left\langle\gamma f\left(x^{*}\right)-A x^{*}, x_{n}-q_{n}\right\rangle+\limsup _{n \rightarrow \infty}\left\langle\gamma f\left(x^{*}\right)-A x^{*}, q_{n}-x^{*}\right\rangle \\
& \quad \leq 0 .
\end{aligned}
$$

Step 8. Finally we prove that

$$
x_{n} \longrightarrow x^{*} .
$$


Indeed, from (3.5), (3.15), and (3.17), we have

$$
\begin{aligned}
\| x_{n}- & x^{*} \|^{2} \\
= & \left\|\alpha_{n}\left(\gamma f\left(u_{n}\right)-A x^{*}\right)+\beta_{n}\left(x_{n}-x^{*}\right)+\left(\left(1-\beta_{n}\right) I-\alpha_{n} A\right)\left(q_{n}-x^{*}\right)\right\|^{2} \\
\leq & \left\|\beta_{n}\left(x_{n}-x^{*}\right)+\left(\left(1-\beta_{n}\right) I-\alpha_{n} A\right)\left(q_{n}-x^{*}\right)\right\|^{2}+2 \alpha_{n}\left\langle\gamma f\left(u_{n}\right)-A x^{*}, x_{n}-x^{*}\right\rangle \\
\leq & {\left[\left\|\left(\left(1-\beta_{n}\right) I-\alpha_{n} A\right)\left(q_{n}-x^{*}\right)\right\|+\beta_{n}\left\|x_{n}-x^{*}\right\|\right]^{2}+2 \alpha_{n} \gamma\left\langle f\left(u_{n}\right)-f\left(x^{*}\right), x_{n}-x^{*}\right\rangle } \\
& +2 \alpha_{n}\left\langle\gamma f\left(x^{*}\right)-A x^{*}, x_{n}-x^{*}\right\rangle \\
\leq & {\left[\left(1-\beta_{n}-\alpha_{n} \bar{\gamma}\right)\left\|\rho_{n}-x^{*}\right\|+\beta_{n}\left\|x_{n}-x^{*}\right\|\right]^{2}+2 \alpha_{n} \gamma h\left\|x_{n}-x^{*}\right\|^{2} } \\
& +2 \alpha_{n}\left\langle\gamma f\left(x^{*}\right)-A x^{*}, x_{n}-x^{*}\right\rangle \\
= & \left(\left(1-\alpha_{n} \bar{\gamma}\right)^{2}+2 \alpha_{n} \gamma h\right)\left\|x_{n}-x^{*}\right\|^{2}+2 \alpha_{n}\left\langle\gamma f\left(x^{*}\right)-A x^{*}, x_{n}-x^{*}\right\rangle .
\end{aligned}
$$

This implies that

$$
\left\|x_{n}-x^{*}\right\|^{2} \leq \frac{2}{2(\bar{\gamma}-\gamma h)-\bar{\gamma}^{2}}\left\langle\gamma f\left(x^{*}\right)-A x^{*}, x_{n}-x^{*}\right\rangle .
$$

Combining (3.61) and (3.69), we obtain that $x_{n} \rightarrow x^{*}$.

This completes the proof of Theorem 3.2.

Corollary 3.3. Let $H, C, f, T, F, A, B, \varphi_{i}, \eta_{i}, K_{i}(i=1,2, \ldots, N)$ be the same as in Theorem 3.2. Let $r_{i}(i=1,2, \ldots, N)$ be a finite family of positive parameter, $\lambda \in(0,2 \alpha],\left\{\alpha_{n}\right\},\left\{\beta_{n}\right\} \subset[0,1]$ and $\left\{t_{n}\right\} \subset(0, \infty)$. If $\mathcal{G}_{1}:=F(\mathcal{C}) \cap M E P(\mathcal{F}) \cap V I(H, B, M) \neq \emptyset$ and conditions (i) and (ii) in Theorem 3.2 are satisfied, then

(1) for each $n \geq 1$ there is a unique $x_{n} \in C$ such that

$$
\begin{aligned}
x_{n}= & \alpha_{n} \gamma f\left(\frac{1}{t_{n}} \int_{0}^{t_{n}} T(s) x_{n} d s\right)+\beta_{n} x_{n} \\
& +\left(\left(1-\beta_{n}\right) I-\alpha_{n} A\right) \frac{1}{t_{n}} \int_{0}^{t_{n}} T(s)\left(P_{C}(I-\lambda B)\right)^{2} \mho^{N} x_{n} d ;
\end{aligned}
$$

(2) the sequence $\left\{x_{n}\right\}$ converges strongly to some point $x^{*} \in \mathcal{G}$, provided that $V_{r_{i}}^{\Theta_{i}}$ is firmly nonexpansive;

(3) $x^{*}$ is the unique solution of variational inequality (3.6). 
Proof. Taking $M=\partial \delta_{C}: H \rightarrow 2^{H}$ in Theorem 3.2, where $\delta_{C}: H \rightarrow[0, \infty)$ is the indicator function of $C$, that is,

$$
\delta_{C}= \begin{cases}0, & x \in C, \\ +\infty, & x \notin C,\end{cases}
$$

then the variational inclusion problem (1.2) is equivalent to variational inequality (1.4), that is, to find $u \in C$ such that

$$
\langle B(u), v-u\rangle \geq 0, \quad \forall v \in C .
$$

Again, since $M=\partial \delta_{C}$, then $J_{M, \lambda}=P_{C}$. Therefore we have

$$
\rho_{n}=P_{C}(I-\lambda B) \xi_{n}, \quad \xi_{n}=P_{C}(I-\lambda B) y_{n}
$$

The conclusion of Corollary 3.3 can be obtained from Theorem 3.2 immediately.

\section{Applications to Optimization Problem}

Let $H$ be a real Hilbert space, $C$ a nonempty closed convex subset of $H, A: H \rightarrow H$ a strongly positive linear bounded operator with a constant $\bar{\gamma}>0$, and $T: C \rightarrow C$ a nonexpansive mapping. In this section we will utilize the results presented in Section 3 to study the following optimization problem:

$$
\min _{x \in F(T)} \frac{1}{2}(\langle A x, x\rangle-h(x))
$$

where $F(T)$ is the set of fixed points of $T$ in $C$ and $h: C \rightarrow R$ is a potential function for $\gamma f$ (i.e., $h^{\prime}(x)=\gamma f(x), x \in C$ ), where $f: C \rightarrow C$ is a contractive mapping with a contractive constant $h \in(0,1)$. We have the following theorem.

Theorem 4.1. Let $H, C, f, T, A$ be the same as above. Let $\left\{\alpha_{n}\right\},\left\{\beta_{n}\right\}$ be sequences in $[0,1]$ satisfying condition (ii) in Theorem 3.2. If $F(T)$ is a nonempty compact subset of $C$, then for each $n \geq 1$ there is a unique $x_{n} \in C$ such that

$$
x_{n}=\alpha_{n} \gamma f\left(T\left(x_{n}\right)\right)+\beta_{n} x_{n}+\left(\left(1-\beta_{n}\right) I-\alpha_{n} A\right) T x_{n}, \quad \forall n \geq 1,
$$

and the sequence $\left\{x_{n}\right\}$ converges strongly to some point $x^{*} \in F(T)$ which is the unique minimal point of optimization problem (4.1).

Proof. Taking $\Theta_{i}=0, \varphi_{i}=0, K_{i}=0, \eta_{i}=0, r_{i}=1(i=1,2, \ldots, N), B=0, \tau=T$ in Corollary 3.3, hence we have $\mathcal{F}=0, V_{r_{i}}^{\Theta_{i}}=I, i=1,2, \ldots, N, y_{n}=\xi_{n}=\rho_{n}=x_{n},\left(1 / t_{n}\right) \int_{0}^{t_{n}} T(s) x_{n} d s=T x_{n}$, for all $n \geq 1, F(\tau)=F(T), \operatorname{MEP}(\mathscr{F})=\operatorname{VI}(H, B, M)=C, \mathcal{G}=F(T)$. Hence from Corollary 3.3 
we know that the sequence $\left\{x_{n}\right\}$ defined by (4.2) converges strongly to some point $x^{*} \in F(T)$ which is the unique solution of the following variational inequality:

$$
\left\langle(A-\gamma f) x^{*}, x-x^{*}\right\rangle \geq 0, \quad x \in F(T) .
$$

Since $T$ is nonexpansive, then $F(T)$ is convex. Again by the assumption that $F(T)$ is compact, therefore it is a compact and convex subset of $C$, and $(1 / 2)(\langle A x, x\rangle-h(x)): C \rightarrow R$ is a continuous mapping. By virtue of the well-known Weierstrass theorem, there exists a point $y^{*} \in F(T)$ which is a minimal point of optimization problem (4.1). As is known to all, (4.3) is the optimality necessary condition [19] for the optimization problem (4.1). Therefore we also have

$$
\left\langle(A-\gamma f) y^{*}, x-y^{*}\right\rangle \geq 0, \quad \forall x \in F(T) .
$$

Since $x^{*}$ is the unique solution of (4.3), we have $x^{*}=y^{*}$.

This completes the proof of Theorem 4.1.

\section{Acknowledgment}

The authors would like to express their thanks to the referees for their valuable suggestions and comments.

\section{References}

[1] S. S. Chang, "Set-valued variational inclusions in Banach spaces," Journal of Mathematical Analysis and Applications, vol. 248, no. 2, pp. 438-454, 2000.

[2] S. S. Chang, "Existence and approximation of solutions for set-valued variational inclusions in Banach space," Nonlinear Analysis: Theory, Methods \& Applications, vol. 47, no. 1, pp. 583-594, 2001.

[3] V. F. Demyanov, G. E. Stavroulakis, L. N. Polyakova, and P. D. Panagiotopoulos, Quasidifferentiability and Nonsmooth Modelling in Mechanics, Engineering and Economics, vol. 10 of Nonconvex Optimization and Its Applications, Kluwer Academic Publishers, Dordrecht, The Netherlands, 1996.

[4] J.-L. Lions and G. Stampacchia, "Variational inequalities," Communications on Pure and Applied Mathematics, vol. 20, pp. 493-519, 1967.

[5] S.-s. Zhang, J. H. W. Lee, and C. K. Chan, "Algorithms of common solutions to quasi variational inclusion and fixed point problems," Applied Mathematics and Mechanics, vol. 29, no. 5, pp. 571-581, 2008.

[6] L.-C. Ceng and J.-C. Yao, "A hybrid iterative scheme for mixed equilibrium problems and fixed point problems," Journal of Computational and Applied Mathematics, vol. 214, no. 1, pp. 186-201, 2008.

[7] S. Li, L. Li, and Y. Su, "General iterative methods for a one-parameter nonexpansive semigroup in Hilbert space," Nonlinear Analysis: Theory, Methods \& Applications, vol. 70, no. 9, pp. 3065-3071, 2009.

[8] S. Saeidi, "Iterative algorithms for finding common solutions of variational inequalities and systems of equilibrium problems and fixed points of families and semigroups of nonexpansive mappings," Nonlinear Analysis: Theory, Methods \& Applications, vol. 70, no. 12, pp. 4195-4208, 2009.

[9] Y. Yao, Y. J. Cho, and R. Chen, "An iterative algorithm for solving fixed point problems, variational inequality problems and mixed equilibrium problems," Nonlinear Analysis: Theory, Methods $\mathcal{E}$ Applications, vol. 71, no. 7-8, pp. 3363-3373, 2009.

[10] W. Kumam and P. Kumam, "Hybrid iterative scheme by a relaxed extragradient method for solutions of equilibrium problems and a general system of variational inequalities with application to optimization," Nonlinear Analysis: Hybrid Systems, vol. 3, no. 4, pp. 640-656, 2009.

[11] V. Colao, G. L. Acedo, and G. Marino, "An implicit method for finding common solutions of variational inequalities and systems of equilibrium problems and fixed points of infinite family of 
nonexpansive mappings," Nonlinear Analysis: Theory, Methods E Applications, vol. 71, no. 7-8, pp. 27082715, 2009.

[12] H. He, S. Liu, and H. Zhou, "An explicit method for finding common solutions of variational inequalities and systems of equilibrium problems and fixed points of an infinite family of nonexpansive mappings," Nonlinear Analysis: Theory, Methods E Applications, vol. 72, no. 6, pp. 31243135, 2010.

[13] C. S. Hu and G. Cai, "Viscosity approximation schemes for fixed point problems and equilibrium problems and variational inequality problems," Nonlinear Analysis: Theory, Methods E Applications, vol. 72, no. 3-4, pp. 1792-1808, 2010.

[14] S. Takahashi and W. Takahashi, "Viscosity approximation methods for equilibrium problems and fixed point problems in Hilbert spaces," Journal of Mathematical Analysis and Applications, vol. 331, no. 1, pp. 506-515, 2007.

[15] S. S. Chang, H. W. J. Lee, and C. K. Chan, "A new method for solving equilibrium problem fixed point problem and variational inequality problem with application to optimization," Nonlinear Analysis: Theory, Methods E Applications, vol. 70, no. 9, pp. 3307-3319, 2009.

[16] P. Dan, Nolinear Mappings of Monotone Type, Sijthoff and Noordhoff, Alphen aan den Rijn, The Netherlands, 1978.

[17] T. Shimizu and W. Takahashi, "Strong convergence to common fixed points of families of nonexpansive mappings," Journal of Mathematical Analysis and Applications, vol. 211, no. 1, pp. 71-83, 1997.

[18] G. Marino and H.-K. Xu, "A general iterative method for nonexpansive mappings in Hilbert spaces," Journal of Mathematical Analysis and Applications, vol. 318, no. 1, pp. 43-52, 2006.

[19] H. K. Xu, "An iterative approach to quadratic optimization," Journal of Optimization Theory and Applications, vol. 116, no. 3, pp. 659-678, 2003. 